\title{
Effect of Cholesterol and Curcumin on Ordering of DMPC Bilayers
}

\author{
Sergey A. Kotenkov ${ }^{1}$ - Oleg I. Gnezdilov ${ }^{1}$ - Aliya V. Khaliullina ${ }^{1,3}$. \\ Oleg N. Antzutkin ${ }^{2} \cdot$ Roustam S. Gimatdinov $^{3}$ · Andrei V. Filippov ${ }^{1,2}$
}

Received: 15 September 2018 / Revised: 12 November 2018 / Published online: 23 November 2018 (c) The Author(s) 2018

\begin{abstract}
In this work, we compared the effects of curcumin and cholesterol directly competing to insert into the DMPC lipid bilayer during bilayer formation from an initially non-ordered state. ${ }^{2} \mathrm{H}$ and ${ }^{14} \mathrm{~N}$ nuclear magnetic resonance spectroscopy showed that curcumin is not embedded deep in the lipid bilayer and interacts mainly with the head group of the lipid. In a more complex system of DMPC/CHOL/CUR, curcumin amplifies the effect of cholesterol on the ordering of lipid acyl chains.
\end{abstract}

\section{Introduction}

Cholesterol (CHOL) is an essential component of mammalian cell membranes. It is embedded in the hydrophobic part of the membrane with its hydroxyl group interacting with the polar head groups of the membrane phospholipids and sphingolipids $[1,2]$. One of the most significant biological properties of CHOL is its ability to increase membrane packing and ordering in the liquid crystalline phase [3-5]. According to measurements of lateral diffusion coefficients of phospholipids and sphingolipids, it has been determined that membrane fluidity decreases as the concentration of CHOL increases [6, 7]. Fluorescent microscopy and ${ }^{2} \mathrm{H}$ nuclear magnetic resonance (NMR) have shown that $\mathrm{CHOL}$ also introduces the liquid ordered phase [8-10].

Curcumin (CUR) is a natural component of the spice Curcuma longa. It is used in the kitchen and actively used in traditional Indian medicine [11, 12]. CUR

Electronic supplementary material The online version of this article (https://doi.org/10.1007/s0072 3-018-1102-2) contains supplementary material, which is available to authorized users.

Andrei V. Filippov

Andrei.Filippov@1tu.se

1 Institute of Physics, Kazan Federal University, 420008 Kazan, Russia

2 Chemistry of Interfaces, Luleå University of Technology, 91187 Luleå, Sweden

3 Kazan State Medical University, 420012 Kazan, Russia 
and its derivatives have anti-oxidant, anti-carcinogenic, anti-mutagenic, antiinflammatory and anti-fungal properties [13-15]. CUR has shown prophylactic and therapeutic capacity for neurodegenerative diseases such as Alzheimer's and Parkinson's, which has been attributed to the compound's interaction with amyloid fibrils [16-18], as well as oligomers and protofibrils [16]. Because of its wide spectrum of pharmacological properties, CUR, such as cholesterol, has been proposed to act on a rather basic biological level, such as on biomembranes. It has been proposed that CUR can regulate the action of membrane proteins indirectly by changing the physical properties of the membrane rather than by directly binding to the proteins [19].

Similarities of CUR and CHOL activity on biomembranes have been suggested in a number of biochemical studies. CUR molecules initially bind to the membrane interface and then at higher concentrations gradually partition to insert into the hydrocarbon region, which has been detected by the binding of CUR with giant unilamellar vesicles [20]. X-ray diffraction experiments have shown that the thickness of DOPC bilayers decreases as a function of the lipid/CUR ratio [21]. Solid-state NMR on magnetically aligned bicelles of DMPC and DSC on bilayers of DMPC and DPPC have shown CUR has a strong effect on membrane structure at low concentrations [22]. CUR inserts deep into the membrane in a transbilayer orientation, anchored through the hydrogen bonding of $-\mathrm{OH}$ groups of phenolic rings of CUR to the phosphate group of lipids and the hydrophobic interaction of the aromatic rings of CUR with phospholipid acyl chains in a manner analogous to that of cholesterol. Like CHOL, CUR binds DMPC in a transbilayer orientation at low concentrations and induces segmental ordering in the lipid membrane [23]. CUR forms higher order oligomeric structures in the membrane that likely span through the bilayer, detected by analysis of the concentration dependence of the order parameter profile derived from NMR results. The apparent abolition of the pretransition with increase in CUR concentration in DMPC and DPPC implies that CUR interacts with the PC head groups [23].

Recently, lateral diffusion on planar lipid multibilayers and ordering of lipids on multibilayered vesicles have been studied by ${ }^{1} \mathrm{H}$ and ${ }^{2} \mathrm{H}$ NMR of DOPC and DMPC at concentrations of CUR varied in the range of $0-40 \mathrm{~mol} \%$ [24]. It has been shown that the presence of CUR in low concentrations $(\sim 8 \mathrm{~mol} \%)$ leads to a moderate decrease in the lateral diffusion coefficients of both saturated and unsaturated lipids, while a further increase in CUR concentration has no effect because of the low solubility of CUR in lipid bilayers. The effect of CUR on ${ }^{2} \mathrm{H}$ NMR quadrupolar splitting is also very small in comparison with that of CHOL. While CUR has a strong effect on the lateral diffusion of DMPC, the same as in DMPC/ CHOL membranes, there is no visible effect of CUR in the presence of CHOL. Therefore, in direct competition to insert into the DMPC lipid bilayer during formation of the bilayer from an initially homogeneous mixture, CHOL overwhelmingly wins and consequently inhibits insertion of curcumin [25].

Despite wide interest in CUR-membrane interactions, studies of the effect of CUR on cholesterol-containing membranes are lacking. In this work, we studied the combined effect of both CUR and CHOL on a lipid bilayer, using ${ }^{2} \mathrm{H}$ and ${ }^{14} \mathrm{~N}$ NMR as methods to study ordering of lipid membranes. 


\section{Materials and Methods}

\subsection{Sample Preparation}

Phospholipids DMPC (dimyristoylphosphatidylcholine) and DMPC with deuterated hydrocarbon chains (DMPC- $\mathrm{d}_{54}$ ) were purchased from Avanti Polar Lipids. DMPC is the typical saturated-chain lipid, commonly used to prepare model lipid bilayers. Curcumin ( $>94 \%$ curcuminoid content, $>80 \%$ CUR) and cholesterol (5-cholesten$3 \beta$-ol) were purchased from Sigma. Structures of DMPC, CUR and CHOL molecules are shown in Fig. 1.

To prepare a vesicular sample, the lipid, cholesterol and curcumin were dissolved in a sufficient amount of ethanol. The ethanol was then evaporated under a constant flow of dry nitrogen, and then the sample was vacuum-pumped overnight to remove solvent traces. The resulting lipid film was hydrated using deionized water at a 5:1 ratio. Five freeze-thaw cycles were applied using liquid nitrogen and warm (323 K) water, according to Traikia et al., which resulted in the formation of a homogeneous vesicular sample [26].

\subsection{1 ${ }^{2} \mathrm{H}$ and ${ }^{14} \mathrm{~N}$ NMR Spectroscopy}

The NMR spectra of DMPC vesicles were recorded in a Bruker AVANCE III (Bruker BioSpin AG, Fällanden, Switzerland) operating at a proton frequency of<smiles>CCCCCCCCCCCCCCCCC(=O)OC[C@H](COP(=O)([O-])OCC[N+](C)(C)C)OC(=O)CCCCCCCC(C)(C)C</smiles>

(B)<smiles>COc1cc(/C=C/C(=O)CC(=O)/C=C/c2ccc(O)c(OC)c2)ccc1O</smiles>

(C)

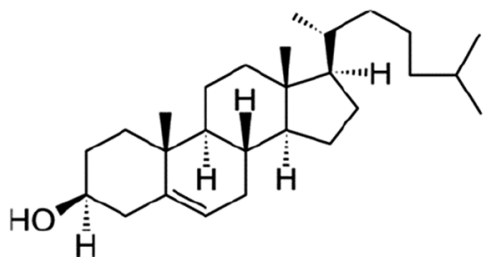

Fig. 1 Chemical structures of: DMPC (a), curcumin (diketo form) (b) and cholesterol (c) molecules 
400.27 MHz applying solid-echo pulse sequence [27] at $308 \mathrm{~K}$. The ${ }^{2} \mathrm{H}$ NMR frequency was $61.422 \mathrm{MHz}$, relaxation delay was $0.25 \mathrm{~s}$, rf-pulse duration was $25 \mu \mathrm{s}$, sweep width was $39 \mathrm{kHz}$ and acquisition time was $0.21 \mathrm{~s}$; a total of $16 \mathrm{k}$ signal transients were collected. ${ }^{14} \mathrm{~N}$ NMR frequency was $28.904 \mathrm{MHz}$; a total of $25 \mathrm{k}$ signal transients were collected.

De-Pake-ing is a method of numerical transformation of unoriented static ${ }^{2} \mathrm{H}$ spectra into a $0^{\circ}$-oriented spectrum [28]. In this case, the segmental order parameters, $\mathrm{S}_{\mathrm{CD}}$, of lipid chains can be calculated $[29,30]$. The ${ }^{2} \mathrm{H}$ spectra were processed by the program NMR-Depaker to generate de-Paked spectra (https://launchpad.net/).

\subsection{2 ${ }^{2} \mathrm{H}$ NMR}

NMR is an appropriate technique for measuring the degree of order in lipid systems $[31,32] .{ }^{2} \mathrm{H}$ nuclei (spin $I=1$ ) have a quadrupole moment, which interacts with the electric field gradient. This interaction can be considered a change in the energy of the Zeeman levels when the nucleus is placed in a strong external magnetic field. Therefore, the equation for the total energy will contain information about how they are oriented relative to each other [33]. The frequency difference between two ${ }^{2} \mathrm{H}$ resonance lines of the $\mathrm{C}-\mathrm{D}$ group, which is experiencing rapid reorientation, is described by the following equation:

$$
\Delta v_{\mathrm{Q}}=\frac{3}{4} \frac{e^{2} q Q}{h}\left(3 \cos ^{2} \theta-1\right) S_{\mathrm{CD}}
$$

where $\left(e^{2} q Q / h\right)$ is the quadrupole coupling constant for the C-D bond $(\sim 167 \mathrm{kHz})$ and $\theta$ is the angle between the bilayer normal and the direction of the external magnetic field, $B_{0}[29,34]$.

The order parameter, $S_{\mathrm{CD}}$, is a function averaged over time. For C-D bonds in a lipid, $S_{\mathrm{CD}}$ is calculated using the following equation:

$$
S_{\mathrm{CD}}=\frac{1}{2} 3 \cos ^{2} \varphi-1,
$$

where $\varphi$ is the angle between the normal to the membrane and the $\mathrm{C}-\mathrm{D}$ bond.

The increase (decrease) value of $S_{\mathrm{CD}}$ for a C-D bond in a lipid bilayer system with axial symmetry is associated with trans-gauche rotational isomerizations of the acyl groups, molecular motions and ordering of lipids in the system [28-30, 33].

\section{Results and Discussion}

\subsection{Lipid Phases and Ordering}

Formation of vesicles in all studied DMPC/CHOL/CUR samples was confirmed by observation of resolved ${ }^{2} \mathrm{H}$ NMR spectra. ${ }^{31} \mathrm{P}$ NMR and ${ }^{2} \mathrm{H}$ NMR experiments performed on vesicles of DMPC- $\mathrm{d}_{54}$ in the presence of CHOL and CUR (Figs. 2 and 


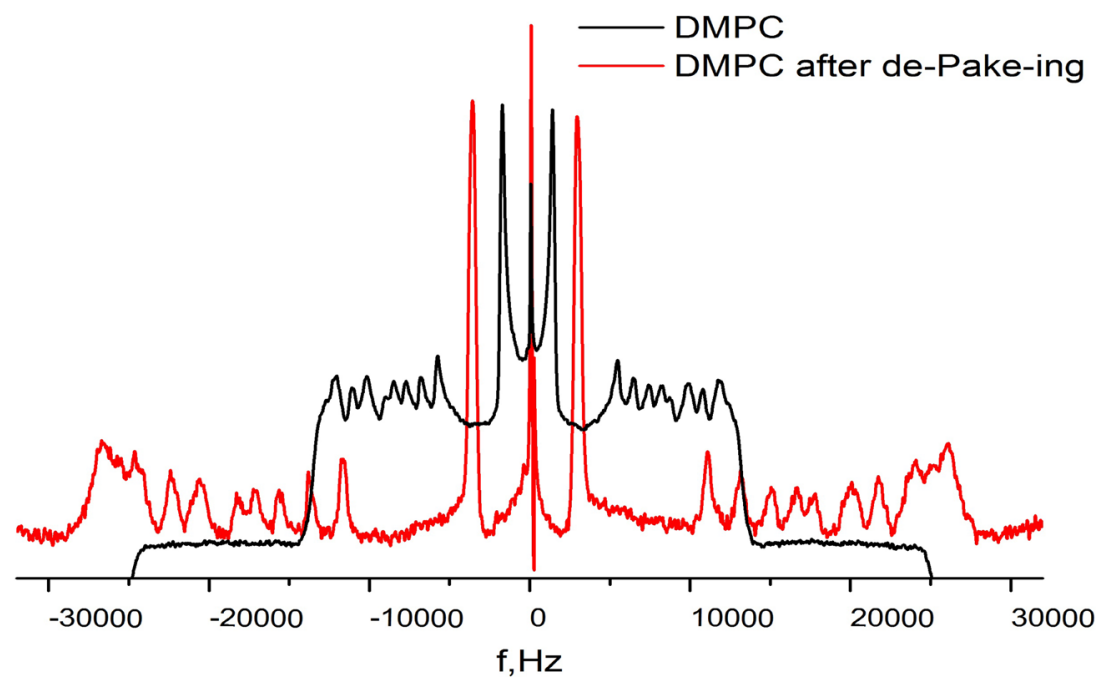

Fig. $2{ }^{2} \mathrm{H}$ NMR spectra of DMPC $\left(\mathrm{d}_{54}\right)$ vesicles before (black line) and after the "de-Pake-ing" processing (red line)

$\mathrm{S} 1, \mathrm{~S} 2$ in ESI) demonstrated that ${ }^{2} \mathrm{H}$ and ${ }^{31} \mathrm{P}$ NMR spectra have shapes typical for the lamellar phase of lipids [31,35].

The ${ }^{2} \mathrm{H}$ spectrum of the control sample of DMPC $\left(\mathrm{d}_{54}\right)$ vesicles shows three distinct resonances. The central resonance line (close to $0 \mathrm{~Hz}$ ) is related to deuterons of water. The two other resonances close to the center of the spectrum refer to the $-\mathrm{CD}_{3}$ terminal groups of the lipid tails. The quadrupole splitting of the methyl group of DMPC is comparable with the data presented in the literature and is equal to $3.2 \mathrm{kHz}$ at $308 \mathrm{~K}$ [36]. Other resonances with the larger splitting correspond to $-\mathrm{CD}_{2}-$ groups in the tail section, situated close to the "heads" of lipids.

The ${ }^{2} \mathrm{H}$ NMR spectrum of lipids with chains enriched with deuterium contains information about ordering of the lipids chains [4, 27]. Figures 3 and S3 in ESI shows a central part of the "de-Pake-ing" ${ }^{2} \mathrm{H}$ NMR spectra of vesicular DMPC- $\mathrm{d}_{54}$ with CUR and CHOL, which is related to the methyl $-\mathrm{CD}_{3}$ groups of the lipid acyl chains.

The presence of curcumin at concentrations of $10 \mathrm{~mol} \%$ increases the quadrupole splitting of the DMPC ${ }^{2} \mathrm{H}$ NMR spectra by ca. $0.25 \mathrm{kHz}$, whereas the concentration of cholesterol in the presence of $15 \mathrm{~mol} \%$ increases the quadrupole splitting of the DMPC ${ }^{2} \mathrm{H}$ NMR spectra by a significantly larger value (by ca. $3.73 \mathrm{kHz}$ ). The mixture of $15 \mathrm{~mol} \% \mathrm{CHOL}$ and $10 \mathrm{~mol} \%$ CUR together increases the quadrupole splitting by ca $4.36 \mathrm{kHz}$. The greatest effect on the quadrupole splitting of the spectra occurs in the mixture, which is caused by the presence of both, cholesterol and curcumin.

Table 1 shows comparisons of the value of the order parameters, calculated by Eq. (1), with $\theta=0$, of the methyl group of the DMPC acyl chain in the presence of curcumin, cholesterol, and a mixture of both. The highest value of the order parameter corresponds to the sample where both cholesterol and curcumin 


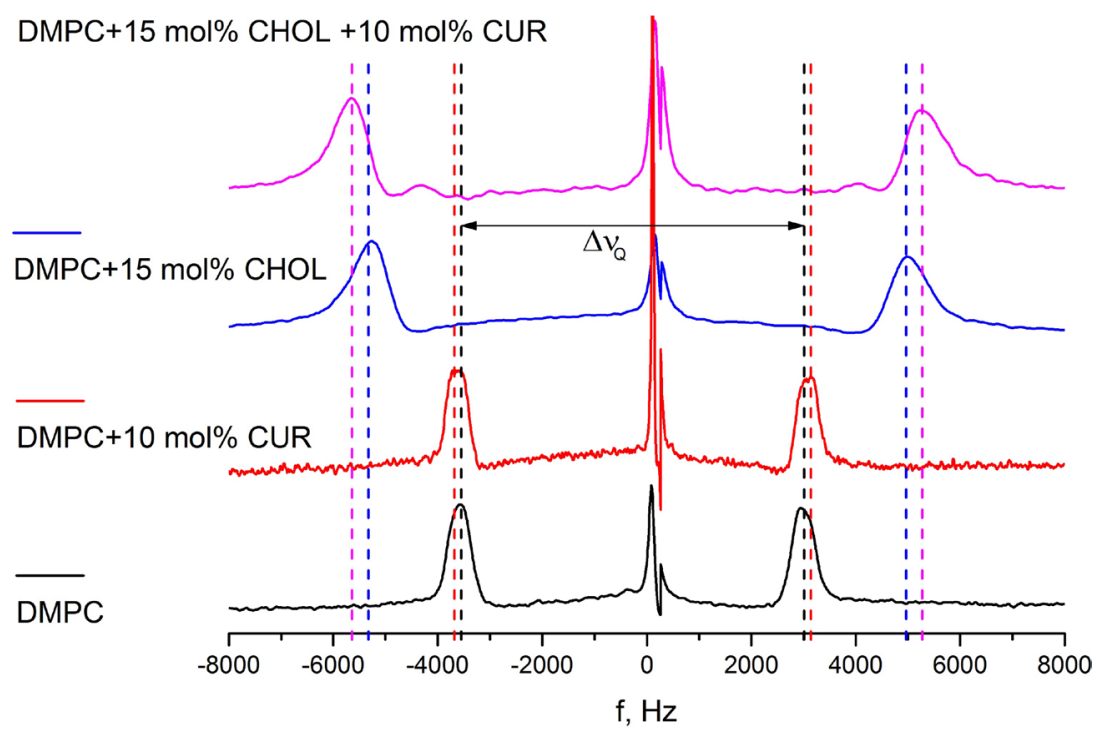

Fig. 3 Comparison of the central part of ${ }^{2} \mathrm{H}$ NMR spectra of pure DMPC- $\mathrm{d}_{54}$, in the presence of $15 \mathrm{~mol} \%$ cholesterol, $10 \mathrm{~mol} \%$ curcumin and a mixture of both in water (with natural ${ }^{2} \mathrm{H}$ content) after de-Pake-ing of all the spectra. $T=308 \mathrm{~K}$

Table 1 Order parameters $S_{\mathrm{CD}}$ for the methyl group of the acyl chains of the lipid DMPC in the presence of curcumin (CUR) and cholesterol (CHOL)

\begin{tabular}{lllll}
\hline $33 \mathrm{~mol} \%$ & $15 \mathrm{~mol} \%$ & $5 \mathrm{~mol} \%$ & $0 \mathrm{~mol} \%$ & $\begin{array}{l}+\mathrm{CHOL} \\
+\mathrm{CUR} \\
(\mathrm{mol} \%)\end{array}$ \\
\hline 0.042 & 0.041 & 0.028 & 0.026 & 0 \\
- & - & 0.030 & 0.027 & 5 \\
0.049 & 0.044 & 0.036 & 0.027 & 10 \\
\hline
\end{tabular}

One (or both) of the components in the mixture is (are) changed [either curcumin (columns) or cholesterol (raws)]

are present. Therefore, in the mixed vesicles, the ordering effect of cholesterol is increased by curcumin.

The difference in the order parameters is probably due to the different areas of interaction of curcumin and cholesterol with lipid molecules in the bilayer. It was shown that the ordering effect of curcumin occurs in the glycerol region of the membrane with the hydrophobic core of the membrane affected to a somewhat lesser degree and this ordering effect is absent at the terminus of the acyl chain [22]. In contrast, profiles of segmental order parameters as a function of acyl chain position indicate that cholesterol inserts deeply into the bilayer and affects all acyl chains [33]. 


\section{$3.2{ }^{14} \mathrm{~N}$ NMR}

Theory and analysis of ${ }^{2} \mathrm{H}$ NMR spectra described in previous sections for the hydrophobic part of DMPC are also valid for ${ }^{14} \mathrm{~N}$ NMR spectra $\left({ }^{14} \mathrm{~N}\right.$ nuclear spin $I=1$ ), but for the hydrophilic "head" region of the phospholipid molecules in vesicles. Figure 4 shows a comparison of the central part of the ${ }^{14} \mathrm{~N}$ NMR spectrum of pure DMPC and spectra of the latter in the presence of curcumin, cholesterol, and a mixture of both. It can be seen that the effect on the quadrupole splitting in the spectra of the hydrophilic part, which includes ${ }^{14} \mathrm{~N}$, of DMPC in the mixture, was caused predominantly by the presence of curcumin, rather than cholesterol.

The ${ }^{14} \mathrm{~N}$ NMR spectra show a small but detectable decrease in the quadrupole splitting, which is indicative of an electrostatic interaction of curcumin with the choline-phosphate hydrophilic part of the DMPC. Interactions of curcumin with the phosphate group oppose the electrostatic interactions between the positively charged choline groups and the negatively charged phosphate groups, which reduce the tetrahedral symmetry for lipids in a membrane and decrease the ${ }^{14} \mathrm{~N}$ quadrupolar splitting [22].

\subsection{Effects of Cholesterol, Curcumin and Both Compounds on DMPC Bilayers}

The effect of cholesterol on biomembranes has been thoroughly studied; therefore, it is reasonable to compare the effects of drug molecules (particularly those with polyphenol groups in their structure) just with CHOL. Experimental studies

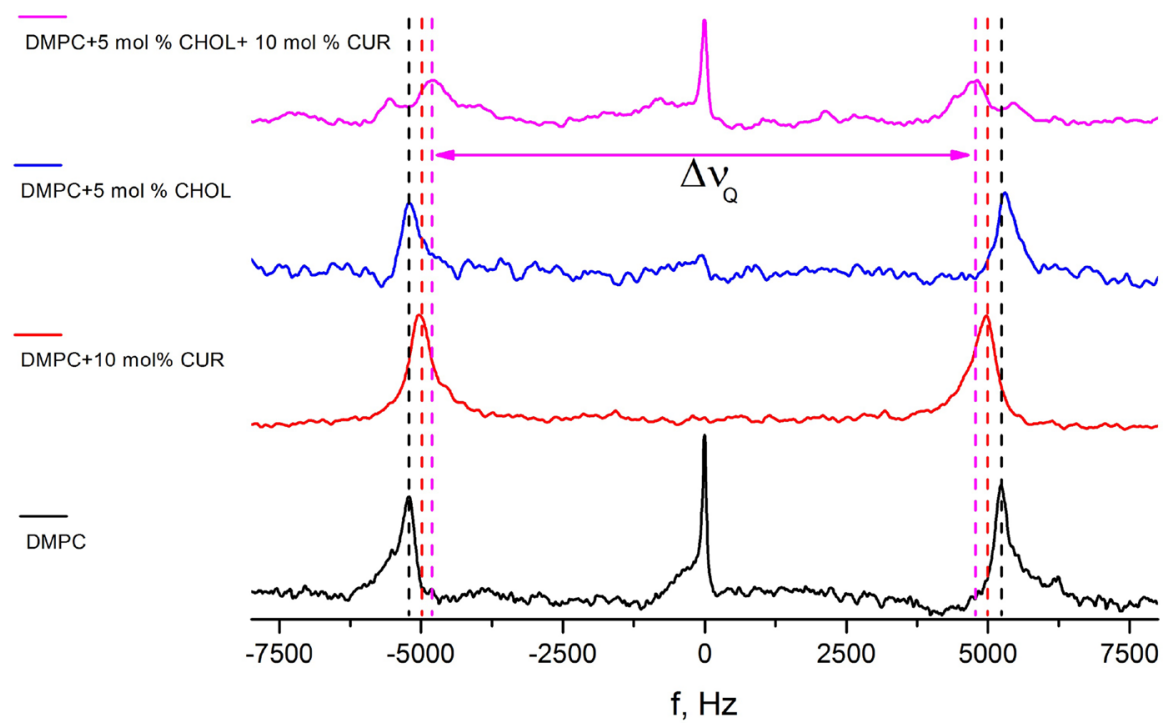

Fig. 4 Comparison of the central part of the ${ }^{14} \mathrm{~N}$ NMR spectra of: (bottom) pure DMPC vesicles and (from bottom to top) DMPC in the presence of $10 \mathrm{~mol} \%$ of curcumin, $5 \mathrm{~mol} \%$ of cholesterol and a mixture of both. $T=308 \mathrm{~K}$ 
and simulations aiming to study the effect of curcumin have been performed on different biomembrane systems and their results are not completely in agreement with each other. The primary place of interaction of CUR with the membrane is the membrane interface (lipid-water interface) near the lipid head group (glycerol) region [37-39], while at higher concentrations, CUR can be located in the lipid membrane hydrophobic region [20,21]. The insertion of CUR in the membrane core is a higher energetic process in comparison with surface adsorption [20], therefore, it can be suggested that a heterogeneous and curved membrane interface may promote insertion of CUR in the membrane hydrophobic core. Inhomogeneity of the water-lipid interface of both vesicles and planar bilayers may also depend on the homogeneity in acyl chain length and type of lipid used to construct the membrane. A recent communication by Tsukamoto et al. [38] showed that CUR localized at the domain's boundary, i.e., the region of a high heterogeneity of the bilayer. Taking these factors into consideration, a higher loading capacity of CUR is expected for vesicles prepared from lipid extracts, while the lowest is expected for planar bilayers. Indeed, vesicles of egg yolk lecithin demonstrated a loading capacity of up to $84 \mathrm{w} / \mathrm{w} \%$ [40], but planar bilayers of DOPC and DMPC did not take more than $10 \mathrm{~mol} \%$ [24]. Insertion of CUR in the bilayer hydrophobic core is also easier to observe in vesicles of lipid extracts $[28,41,42]$ and synthetic lipids [22], but in the case of planar bilayers of synthetic lipids, CUR at higher concentrations tends to aggregate near the hydrophobic interface [24].

In a number of publications, the effect of CUR has been compared with that of CHOL. Of course, these comparisons were qualitative and concern only some effects, such as packing of lipids acyl chains (ordering of the chains), and CUR molecule orientation in the bilayer. In this study, we have had a rare chance to compare CUR and CHOL through their direct competition to insert into the DMPC lipid bilayer during its formation from an initially homogeneous mixture. It is evident that CHOL overwhelmingly wins. The interaction of CHOL with DMPC acyl chains is stronger compared to the effect of curcumin. Insertion of CUR in lipid bilayers is a high energetic process in comparison with the adsorption of CUR on the lipid surface, even in bilayers without CHOL [20, 21]; in more tightly packed DMPC/CHOL membranes, it might take even more energy and become less probable. We suggest a model (see Fig. 5), in which the head group of the lipid interacts with curcumin, and the acyl chain with cholesterol. The lipid molecules are "bound" cholesterol and curcumin, top and bottom.

The overwhelming effect of CHOL over CUR probably has biological relevance. Indeed, if CUR and other polyphenolic drugs just slightly modulate the biological membrane surface (or just properties of surface of the membrane), this may not disturb the structure and essential functions of living cells. In addition, there is a possibility that the curcumin increases binding of cholesterol in the cellular membrane, thereby preventing cholesterol release into the blood stream. This could explain the lowering effect of curcumin against hypercholesterolemia [43]. Further study of interaction between CUR and CHOL embedded in lipid bilayers might shed some light on this aspect. 


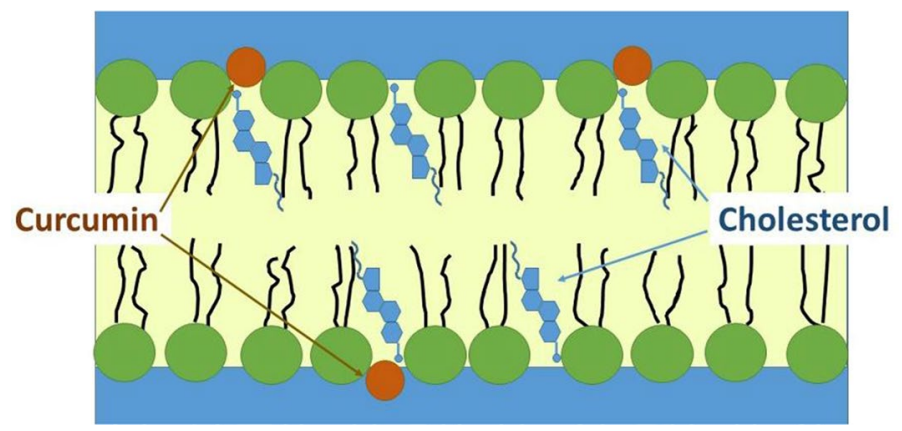

Fig. 5 Proposed model of the interaction of the lipid DMPC with curcumin and cholesterol

\section{Conclusion}

We conclude according to ${ }^{2} \mathrm{H}$ and ${ }^{14} \mathrm{~N}$ NMR spectroscopy that curcumin is not embedded deep within the lipid bilayer and interacts mainly with the head group of the lipid. In a more complex system of DMPC/CHOL/CUR, curcumin increases the effect of cholesterol on the ordering of lipid acyl chains.

Acknowledgements We are grateful to the Kempe Foundation in memory of J.C. and Seth M. Kempe and LTU laboratory fund, which provided a few grants that funded the purchase of the NMR equipment. NMR measurements were also partly carried out on the equipment of the Federal Centre of Collective Facilities of Kazan Federal University.

Open Access This article is distributed under the terms of the Creative Commons Attribution 4.0 International License (http://creativecommons.org/licenses/by/4.0/), which permits unrestricted use, distribution, and reproduction in any medium, provided you give appropriate credit to the original author(s) and the source, provide a link to the Creative Commons license, and indicate if changes were made.

\section{References}

1. A.M. Smondyrev, M.L. Berkowitz, Biophys. J. 77, 2075-2089 (1999)

2. M. Pasenkiewicz-Gierula, T. Róg, K. Kitamura, A. Kusumi, Biophys. J. 78, 1376-1389 (2000)

3. R.A. Haberkorn, R.G. Griffin, M.D. Meadows, E. Oldfield, J. Am. Chem. Soc. 99, 7353-7355 (1977)

4. E. Oldfield, M. Meadows, D. Rice, R. Jacobs, Biochemistry 17, 2727-2740 (1978)

5. E. Falck, M. Patra, M. Karttunen, M.T. Hyvönen, I. Vattulainen, J. Chem. Phys. 121, 1267612689 (2004)

6. A. Filippov, G. Orädd, G. Lindblom, Biophys. J. 84, 3079-3086 (2003)

7. A. Filippov, G. Orädd, G. Lindblom, Langmuir 19, 6397-6400 (2003)

8. S.L. Veatch, S.L. Keller, Phys. Rev. Lett. 89, 2681011-2681014 (2002)

9. G. Lindblom, G. Orädd, A. Filippov, Chem. Phys. Lipids 141, 179-184 (2006)

10. A. Filippov, G. Orädd, G. Lindblom, Biophys. J. 93, 3182-3190 (2007)

11. B. Joe, M. Vijayakumar, B.R. Lokesh, Crit. Rev. Food Sci. Nutr. 44, 97-111 (2004)

12. A. Goel, A.B. Kunnumakkara, B.B. Aggarwal, Biochem. Pharmacol. 75, 787-809 (2008) 
13. L.V. Darbinyan, L.E. Hambarazumyan, K.V. Simonyan, V.A. Chavushyan, L.P. Manukyan, S.A. Badalyan, N. Khalaji, V.H. Sarkisian, Metab. Brain Dis. 32, 1791-1803 (2017)

14. D.J. Bound, P.S. Murthy, P. Srinivas, Food Chem. 210, 371-380 (2016)

15. W. Lee, D.G. Lee, IUBMB Life 66, 780-785 (2014)

16. X. Zhang, Y. Tian, Z. Li, X. Tian, H. Sun, H. Liu, A. Moore, C. Ran, J. Am. Chem. Soc. 135, 16397-16409 (2013)

17. Y. Masuda, M. Fukuchi, T. Yatagawa, M. Tada, K. Takeda, K. Irie, K. Akagi, Y. Monobe, T. Imazawa, K. Takegoshi, Bioorg. Med. Chem. 19, 5967-5974 (2011)

18. K. Liu, T.L. Guo, J. Chojnacki, H.G. Lee, X. Wang, S.L. Siedlak, W. Rao, X. Zhu, S. Zhang, A.C.S. Chem, Neurosci. 3, 141-146 (2012)

19. H.I. Ingolfsson, R.E. Koeppe, O.S. Andersen, Biochemistry 46, 10384-10391 (2007)

20. Y. Sun, C.-C. Lee, W.-C. Hung, F.-Y. Chen, M.-T. Lee, H.W. Huang, Biophys. J. 95, 2318-2324 (2008)

21. W.-C. Hung, F.-Y. Chen, C.-C. Lee, Y. Sun, M.-T. Lee, H.W. Huang, Biophys. J. 94, 4331-4338 (2008)

22. J. Barry, M. Fritz, J.R. Brender, P.E.S. Smith, D.K. Lee, A. Ramamoorthy, J. Am. Chem. Soc. 131, 4490-4498 (2009)

23. G.F. Chen, Y.Y. Chen, N.N. Yang, X.J. Zhu, L.Z. Sun, G.X. Li, Sci. China Life Sci. 55, 527-532 (2012)

24. A.V. Filippov, S.A. Kotenkov, B. Munavirov, O.N. Antzutkin, Langmuir 30, 10686-10690 (2014)

25. A.V. Filippov, S.A. Kotenkov, B.V. Munavirov, A.V. Khaliullina, O.I. Gnezdilov, O.N. Antzutkin, Mendeleev Commun. 26, 109-110 (2016)

26. M. Traïkia, D.E. Warschawski, M. Recouvreur, J. Cartaud, P.F. Devaux, Eur. Biophys. J. 29, 184195 (2000)

27. J.H. Davis, K.R. Jeffrey, M. Bloom, M.I. Valic, T.P. Higgs, Chem. Phys. Lett. 42, 390-394 (1976)

28. M. Bloom, J.H. Davis, A.L. Mackay, Chem. Phys. Lett. 80, 198-202 (1981)

29. J.H. Davis, Biophys. J. 27, 339-358 (1979)

30. M. Ouellet, G. Bernard, N. Voyer, M. Auger, Biophys. J. 90, 4071-4084 (2006)

31. J. Seelig, Q. Rev, Biophys. 10, 353-418 (1977)

32. M. Lafleur, B. Fine, E. Sternin, P.R. Cullis, M. Bloom, Biophys. J. 56, 1037-1041 (1989)

33. M. F. Brown, S. Lope-Piedrafita, G. V Martinez, H. I. Petrache, in: Modern Magnetic Resonance, G.A. Webb (ed.) (Springer, Dordrecht, 2006) pp. 249-260

34. S. Persson, J.A. Killian, G. Lindblom, Biophys. J. 75, 1365-1371 (1998)

35. J. Schiller, M. Müller, B. Fuchs, K. Arnold, D. Huster, Curr. Anal. Chem. 3, 283-301 (2007)

36. J.P. Douliez, A. Léonard, E.J. Dufourc, J. Phys. Chem. 100, 18450-18457 (1996)

37. H.I. Ingólfsson, P. Thakur, K.F. Herold, E.A. Hobart, N.B. Ramsey, X. Periole, D.H. De Jong, M. Zwama, D. Yilmaz, K. Hall, T. Maretzky, H.C. Hemmings, C. Blobel, S.J. Marrink, A. Koçer, J.T. Sack, O.S. Andersen, ACS Chem. Biol. 9, 1788-1798 (2014)

38. M. Tsukamoto, K. Kuroda, A. Ramamoorthy, K. Yasuhara, Chem. Commun. (Camb.) 50, 34273430 (2014)

39. W. W. Kopec̈, J. Telenius, H. Khandelia, FEBS J. 280, 2785-2805 (2013)

40. A. Karewicz, D. Bielska, B. Gzyl-Malcher, M. Kepczynski, R. Lach, M. Nowakowska, Colloids Surfaces B Biointerfaces. 88, 231-239 (2011)

41. Y. Niu, X. Wang, S. Chai, Z. Chen, X. An, W. Shen, J. Agric. Food Chem. 60, 1865-1870 (2012)

42. A. Kunwar, A. Barik, R. Pandey, K.I. Priyadarsini, Biochim. Biophys. Acta-Gen. Subj. 1760, 1513-1520 (2006)

43. S.A. Hussein, Y.A. El-Senosi, M.R. Ragab, M.M.F. Hammad, Benha Veterinary. Med. J. 27, 277289 (2014) 